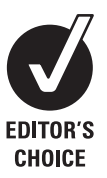

Correspondence to:
Dr Jeremy S Lewis,

PhD, Consultant Physiotherapist,

Therapy Department, Chelsea

and Westminster NHS

Healthcare, 369 Fulham Road,

London SW10 9NH, UK; jeremy.

lewis@chelwest.nhs.uk

Accepted 15 September 2008

Published Online First

17 October 2008

\section{Rotator cuff tendinopathy/subacromial impingement syndrome: is it time for a new method of assessment?}

\author{
J S Lewis
}

\begin{abstract}
Disorders of the shoulder are extremely common, with

reports of prevalence ranging from $30 \%$ of people experiencing shoulder pain at some stage of their lives up to $50 \%$ of the population experiencing at least one episode of shoulder pain annually. In addition to the high incidence, shoulder dysfunction is often persistent and recurrent, with $54 \%$ of sufferers reporting ongoing symptoms after 3 years. To a large extent the substantial morbidity reflects (i) a current lack of understanding of the pathoaetiology, (ii) a lack of diagnostic accuracy in the assessment process, and (iii) inadequacies in current intervention techniques. Pathology of the rotator cuff and subacromial bursa is considered to be the principal cause

a clinical hypothesis attempting to explain the cause of presenting symptoms rather than to a definitive diagnosis based on robust and definitive biomechanical, histological and imaging evidence to explain the basis of the presenting symptoms.

The substantial morbidity associated with rotator cuff pathology reflects (i) a current lack of understanding of the pathoaetiology, (ii) a lack of diagnostic accuracy in the assessment process, and (iii) inadequacies in current intervention techniques. The dilemmas associated with current practice are discussed and an alternative method for the clinical examination of the shoulder for this group of patients is proposed.
\end{abstract} of pain and symptoms arising from the shoulder. Generally these diagnostic labels relate more to a clinical hypothesis as to the underlying cause of the symptoms than to definitive evidence of the histological basis for the diagnosis or the correlation between structural failure and symptoms.

Diagnosing rotator cuff tendinopathy or subacromial impingement syndrome currently involves performing a structured assessment that includes taking the patient's history in conjunction with performing clinical assessment procedures that generally involve tests used to implicate an isolated structure. Based on the response to the clinical tests, a diagnosis of rotator cuff tendinopathy or subacromial impingement syndrome is achieved. The clinical diagnosis is strengthened with the findings from supporting investigations such as blood tests, radiographs, ultrasound, magnetic resonance imaging (MRI), computed axial tomography (CT), radionucleotide isotope scan, single photon emission computed tomography, electromyography, nerve conduction and diagnostic analgesic injection. This process eventually results in the formation of a clinical hypothesis, and then, in conjunction with the patient, a management plan is decided upon and implemented.

This paper focuses on the dilemmas associated with the current process, and an alternative method for the clinical examination of the shoulder for this group of patients is proposed.

Pathology of the rotator cuff and subacromial bursa is considered to be the principal cause of pain and symptoms arising from the shoulder. This is reflected in the variety of diagnostic labels given to describe the pathology: rotator cuff tendinopathy/ tendinosis/tendinitis, supraspinatus tendinopathy/ tendinosis/tendinitis, subacromial impingement syndrome, subacromial bursitis, bursal reaction, partial thickness, full thickness and massive rotator cuff tear. Generally these diagnostic labels relate to

\section{ASSESSMENT OF ROTATOR CUFF PATHOLOGY}

Historically, musculoskeletal assessment of the shoulder has been based around a premise that it is possible to isolate individual structures and apply a mechanical procedure that either compresses or stretches the tissue of interest or requires it to contract. However, it is unlikely that any test would not stretch or compress adjacent structures or cause them to contract during the procedure. Without doubt this is one of the reasons why more recent studies on the sensitivity, specificity and predictive accuracy of tests ${ }^{1-4}$ have concluded that, although they have a high sensitivity and reproduce symptoms, they have an associated low specificity which substantially reduces their utility in deriving a specific diagnosis. As such the commonly used orthopaedic special tests should be thought of as pain or symptom provocation tests, without the ability to contribute to a structural diagnosis.

Examples of clinical tests used to identify structural pathology in current use include the O'Brien active compression test for superior labral pathology, ${ }^{5}$ the posterior capsule length test to assess the extensibility of the posterior glenohumeral capsule and Jobe's "supraspinatus test" to assess the strength and pain response from the supraspinatus musculotendinous unit.' Other tests include the Neer sign, ${ }^{8}$ which has been embraced with other tests, such as the Hawkins' test ${ }^{9}$ and the Internal Rotation Resistance Stress Test,$^{10}$ as clinical methods to implicate the acromion as the cause of the presenting shoulder symptoms.

There are at least three reasons why the clinical assessment procedures for rotator cuff tendinopathy/impingement cannot isolate individual tendons and other structures and inform an accurate diagnosis. These are the morphology of the rotator cuff, the position and innervation of the subacromial 
bursa $(\mathrm{SAB})$, and the lack of correlation between symptoms and contemporary methods of imaging.

The rotator cuff tendons do not function as separate entities. ${ }^{11}$ The clinical implications of the confluence of tendon, ligament (coracohumeral) and glenohumeral capsule are profound and clinical tests proposed to identify symptoms arising from any one individual tendon ${ }^{12}{ }^{13}$ are groundless. The lack of specificity of the clinical tests for the rotator cuff has been demonstrated ${ }^{14}{ }^{14}$ and clinical reasoning based on the response of these tests needs to be re-evaluated. In addition to the fusing of the rotator cuff tendons, the large $S A B$ is innervated ${ }^{15} 16$ and appears to have a central role in the presentation of pain in the shoulder. ${ }^{15-20}$ Any test designed to assess the integrity and pain response from any of the rotator cuff tendons would involve bursal tissue. Two comprehensive systematic reviews of the diagnostic accuracy of the rotator cuff tests have been conducted ${ }^{14}$ and have reached similar conclusions. Both reviews reported that, although a myriad of clinical tests have been proposed to selectively assess the rotator cuff and subacromial bursal tissues and the integrity of the subacromial space, no test or series of tests have emerged that are capable of doing this.

The ability to achieve an accurate structural diagnosis is further challenged by the poor correlation between radiological imaging and symptoms. Using MRI, Frost $e t a^{21}$ reported that structural pathology in the rotator cuff in 42 individuals with a clinical diagnosis of subacromial impingement syndrome was similar to that in 31 age-matched asymptomatic individuals. Similar findings have been reported by others investigating the relationship between radiograms, diagnostic ultrasound (US) and MRI, and symptoms. ${ }^{22-26}$

A series of investigations involving analgesic injections for shoulder pain and weakness have concluded that the perceived weakness during clinical strength testing of the rotator cuff may be better explained as pain inhibition than as a result of structural failure. ${ }^{27-29}$ The role of the rotator cable may help to explain why tears of the rotator cuff identified in imaging investigations may not relate to a loss of shoulder function. The tendon tissue medial to the cable may act through the cable to produce normal shoulder movement even in the presence of structural failure of the rotator cuff located lateral to the cable. This requires ongoing investigation.

The gold standard for diagnostic comparison with the clinical tests has traditionally been direct intraoperative observation, or indirect methods such as MRI or US. As not all structural failure correlates with symptoms, ${ }^{21-26}$ it is apparent that these gold standard tests are not ideal reference tests, and this explains the high numbers of false negatives and false positives reported in these investigations. Of concern, clinical decision-making based upon practice that combines combinations of the currently recommended clinical tests supported by information gained from imaging or observational findings is potentially flawed and does not provide a robust framework for the clinician to clinically reason the structure(s) involved in the symptomatology and create a credible management plan. Smidt and Green ${ }^{30}$ have acknowledged that the reproducibility and validity of the diagnosis and classification system for shoulder complaints are inadequate. As such, the clinical reality is that a new method of assessment needs to be considered.

Difficulties in deriving a definitive structural diagnosis of symptomatic shoulder tissues have been recognised by others. $^{1341431-33}$ In response to these difficulties, alternative methods of assessment have been suggested. ${ }^{34}{ }^{35}$ These methods do not reject traditional methods of shoulder assessment but recognise their inability to achieve a definitive diagnosis due to low specificities. The poor specificities and inadequate likelihood ratios reduce the ability of these tests to inform the clinical decision-making process and patient management. Clinicians are increasingly aware of the limitations of current assessment procedures in the shoulder ${ }^{12414}$ and alternative testing methods have been proposed. ${ }^{34}$ However, these procedures require rigorous assessment of their reliability and validity before being adopted.

\section{THE SHOULDER SYMPTOM MODIFICATION PROCEDURE}

The current proposal advocates the use of special orthopaedic tests only as pain or symptom-provoking procedures and that these procedures should be used clinically in conjunction with other physical movements identified by the patient that reproduce their shoulder pain. Once the movement or activity that reproduces the shoulder symptoms has been agreed upon, the Shoulder Symptom Modification Procedure (SSMP) is applied. The SSMP is a series of four mechanical techniques that are applied while the patient performs the activity or movement that most closely reproduces the symptoms experienced by the patient (fig 1 ). The reason these procedures may reduce symptoms is not known. It could be due to soft tissue or joint displacement, changing sensory motor control or neuromodulation.

The starting point is to select a movement or activity that reproduces the patient's symptoms. This may be a movement identified by the patient or one taken from the series of currently recommended clinical orthopaedic tests that most closely correlates with the patient's symptoms. Information from the patient's history will help to identify the most relevant position in which to examine the patient. For example, a freestyle swimmer complaining of shoulder pain during the pull through phase of swimming should be tested in prone and a tennis player experiencing shoulder pain during a backhand stroke should be tested in standing while performing this movement. Weights, sporting equipment, vocational tools and resistance tubing may be used to reproduce the symptoms if the free active movement proves insufficient. Any contraindications to movement and testing must be observed. Once the provocative activity has been identified, the SSMP process is commenced with the aim of identifying one or a series or techniques that reduce symptoms, by either decreasing pain and/or increasing movement. Examples of this type of assessment are already strongly entrenched in clinical practice to determine whether structures associated with the spine influence shoulder symptoms. ${ }^{36}{ }^{37}$ The SSMP involves four principal procedures. However, additional techniques may be added to the four fundamental tests. The starting procedure and the order of testing are changeable. A brief description of each technique within the SSMP is as follows.

\section{Humeral head procedure}

This component of the SSMP aims to influence the humeral head position in relationship to the glenoid fossa. These procedures involve a series of pressure/displacement techniques and muscle contraction techniques. The pressure techniques are applied in the region of the humeral head and include the use of manual pressure, mobilisation belts, resistance tubing or neoprene slings around the humeral head. Anteriorly or posteriorly directed pressure is maintained during the patient's aggravating activity. Neoprene is preferred due to its comfort and the ability to modify the anterior or posterior force with a medial, lateral, inferior and/or superior force to determine 


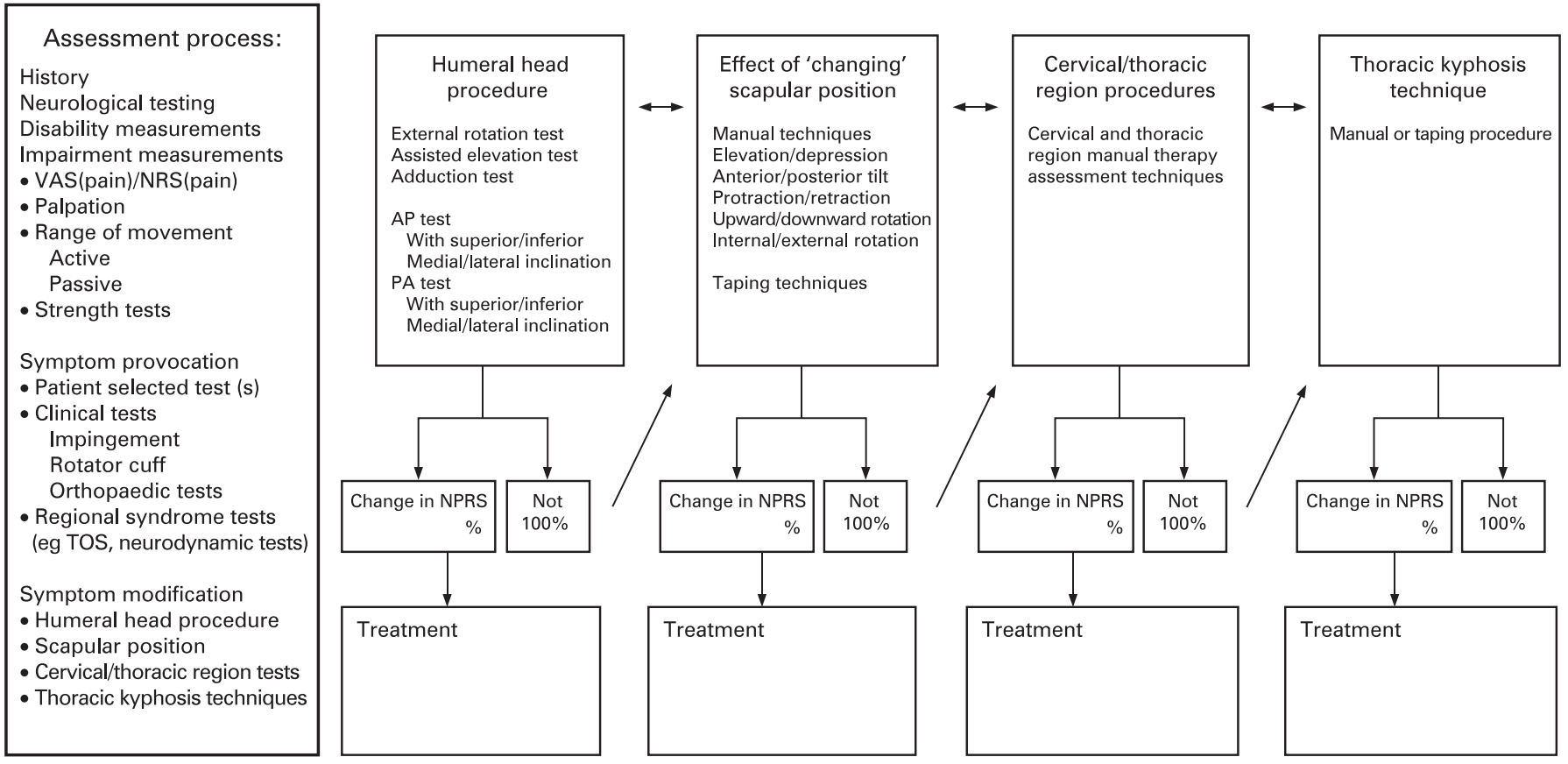

Abbreviations: AP (anterior-posterior), PA (posterior-anterior), VAS (visual analogue scale), NPRS (numerical pain rating scale), TOS (thoracic outlet syndrome)

Clinical reasoning and management plan:

Patients' name, date of birth and identification

Clinician's name, signature and date

Figure 1 The shoulder symptom modification procedure.

which position has the most beneficial effect on symptoms (fig 2).

Muscle contraction procedures involve resisted movements that modify muscle activity at the shoulder, altering the pattern of shoulder muscle contractions that may influence symptoms. Examples include repeating the aggravating activity during a sustained contraction of the shoulder external rotators, humeral head depressors and/or adductors. In this instance, lightweight resistance with rubber tubing is used to create the muscle contraction during the movement to determine its effect on symptoms (fig 3). Procedures that may influence the humeral head position via muscle contraction may be relevant in managing symptoms. Superior translation of the humeral head on the glenoid fossa is observed in patients with subacromial impingement syndrome and rotator cuff disease, ${ }^{38} 39$ and procedures that activate humeral head depressors may counter this translation. ${ }^{40}$ If a particular movement results in the complete cessation of symptoms then the testing process is over. If there is only a partial reduction in symptoms the amount is noted and other components of the SSMP are tested to determine whether a better response is achieved from another technique or whether a combination of techniques results in a greater reduction of symptoms.

\section{Changing scapular position}

A series of manual techniques to slightly modify the scapular position are applied to see whether symptoms change during the aggravating movement. The changes made to the scapular position should be relatively small. If manual techniques are not possible, the application of tape to facilitate a change in scapular position may be considered. ${ }^{35}$ The changes may be in one plane of movement or in combinations of planes of movement.

\section{Cervical and thoracic region procedures}

Structures within the cervical and thoracic region may refer pain to the shoulder. A variety of procedures, often described as muscle, soft tissue, osseous and joint-based techniques, should be applied to determine their effect on the patient's aggravating movement. The effect of changing the thoracic kyphosis is also investigated. The patient's thoracic kyphosis is reduced either through gentle manual reduction or via taping (fig 4). Once this is achieved the aggravating activity is retested to determine the response. Taping the thoracic region and shoulder does not appear to have a placebo effect on pain and range of shoulder movement. ${ }^{35}$

Although the order of the techniques used in the SSMP is interchangeable, two important aspects need to be considered. First, the magnitude of the change in pain experienced by the patient as a result of one or a combination of techniques during the SSMP process should ideally be a minimum of 30\% change from the baseline pain using a numerical pain rating scale (NPRS). Although any single SSMP or combination of SSMPs that both the patient and clinician agree on can be used, a $30 \%$ change represents a substantial and meaningful change for patients. ${ }^{4142}$ Second, there should be consistency of response to the SSMPs that have been selected. There should be a similar 

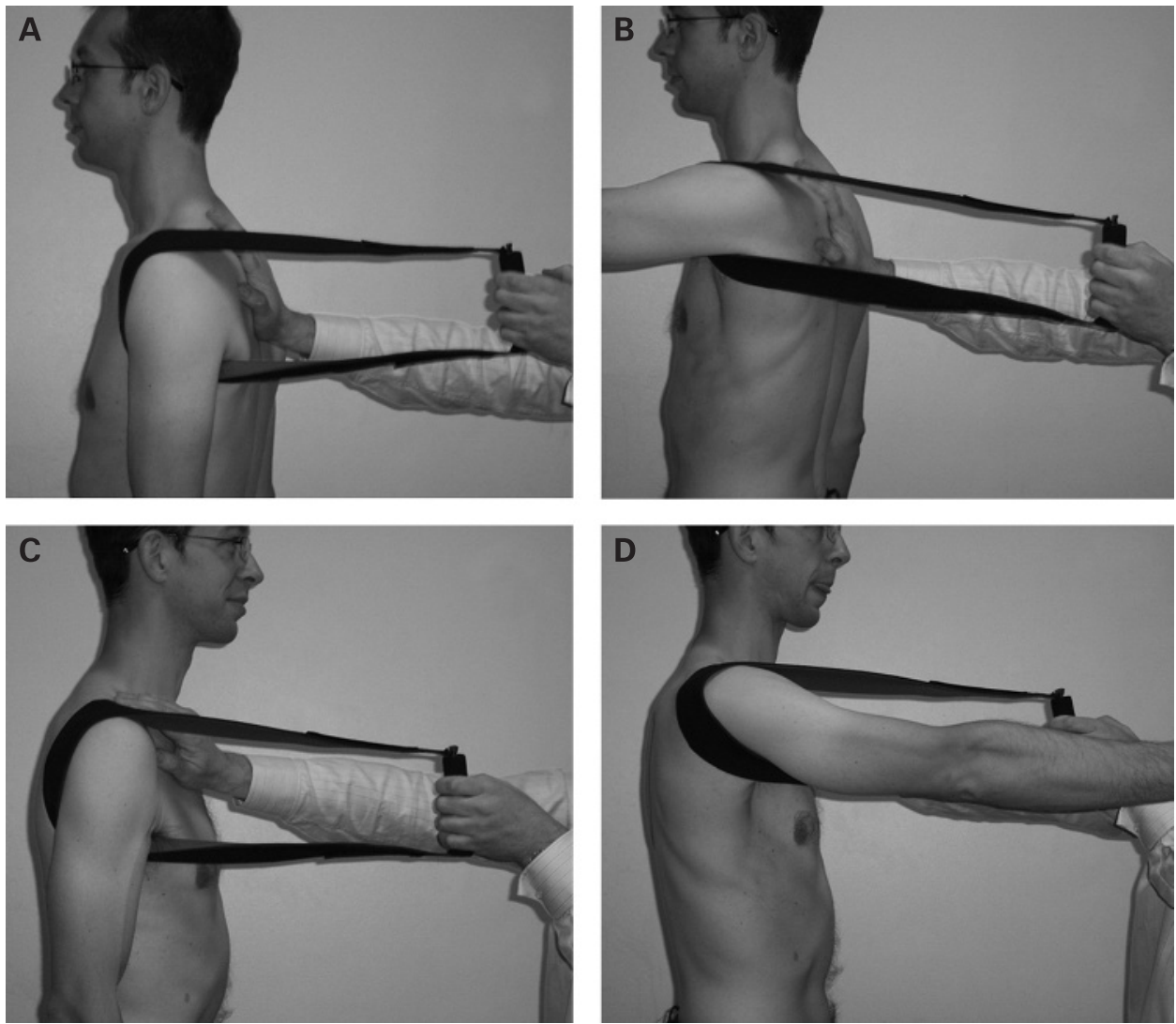

Figure 2 One example of a "humeral head" procedure.

change in symptoms with the SSMP when tested at least twice within the assessment session, with the response being consistent on both occasions. This will increase both the patient's and the clinician's confidence in the process.

Once the clinician and patient agree on the SSMPs that have resulted in the greatest reduction in symptoms, a rehabilitation programme based on these responses is formulated. It is beyond the scope of this paper to discuss treatment options, but in general they include techniques that are similar to the testing procedures. If the SSMP process does not produce any change, the clinician should consider other treatment options such as injection therapy, pain-relieving techniques, general or specific shoulder rehabilitation exercise programmes, modalities, taping or surgery.
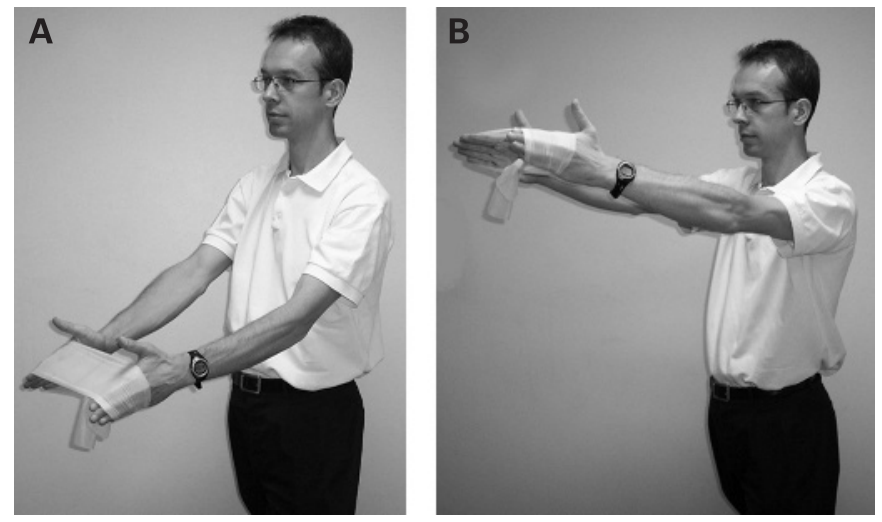

Figure 3 One example of a technique to assess the influence of altered muscle activation on shoulder movement.

\section{EVIDENCE TO SUPPORT THIS MODEL}

The SSMP is not meant as a replacement for current clinical practice but as an adjunct to help support the clinical decisionmaking process by identifying factors that alleviate the patient's shoulder symptoms. Some evidence exists to support this model. Lewis et $a^{35}$ used taping as a method of changing the thoracic kyphosis and scapular position and reported that, when compared with no tape or a placebo taping procedure, the postural correction taping led to an increase in shoulder movement in people with and without shoulder symptoms. Other investigations have also reported that a reduction in the thoracic kyphosis leads to an increase in shoulder movement. ${ }^{43}$ Similarly, in patients with shoulder pain, posteriorly directed pressure applied to the region of the humeral head led to an immediate increase in shoulder elevation range and concomitant decrease in pain when compared with a sham and a control technique. ${ }^{44}$ Boyle ${ }^{45}$ reported that manual therapy techniques in the upper thoracic region substantially reduced pain in a case series of two patients presenting with impingement syndrome. Although these examples are limited, they suggest that a variety of techniques may influence shoulder symptoms in patients who present with a clinical presentation of rotator cuff tendinopathy/subacromial impingement.

\section{ADVANTAGES OF THIS MODEL}

A definitive clinical diagnosis of rotator cuff tendinopathy and subacromial impingement cannot currently be made. Clinicians treating low back pain have recognised the complexity of making a definitive diagnosis, ${ }^{46}$ and terms such as non-specific low back pain have been used in research investigations. ${ }^{47}$ The same diagnostic dilemma faces the clinician when examining a 

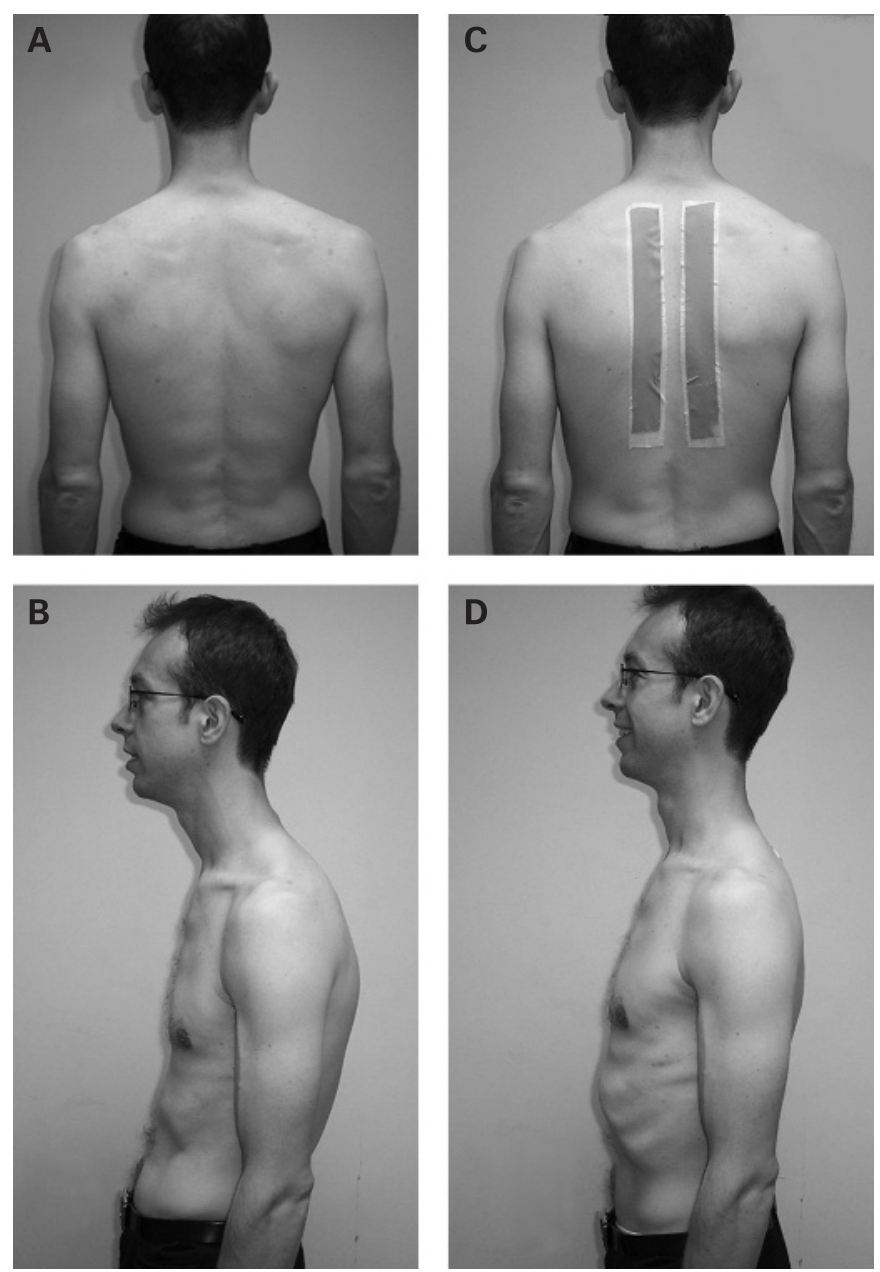

Figure 4 One example of a thoracic kyphosis modification procedure.

patient with shoulder pain, and a more appropriate diagnosis for rotator cuff tendinopathy/subacromial impingement may be mechanical shoulder pain. As we gain a better understanding of the aetiology and pathology, clinical assessment techniques will improve in their ability to delineate reliable, valid and robust clinical diagnostic categories.

The SSMP recognises the inadequacies of the present method of assessment and proposes that patient management may be guided by the response to symptom modification. These assessment procedures may be applied sequentially with one building upon the previous until the most positive single procedure or combination of procedures has been identified. The assessment procedures are then used as treatment techniques for the management of the patient's condition. For example, a reduction in the thoracic kyphosis may result in a partial reduction in pain and partial improvement in shoulder flexion range, and if, in addition to this, an anteriorly to posteriorly directed pressure on the humeral head results in total alleviation of pain and restoration of full range of movement then the rehabilitation programme may include exercises to decrease the thoracic kyphosis together with shoulder movement activities performed while an anterior-to-posterior pressure in the region of the humeral head is applied. The programme is graduated according to the individual's needs and aims to restore normal function.

\section{What is already known on this topic}

The lack of specificity of the clinical tests for the rotator cuff and subacromial impingement has been demonstrated in a number of research investigations and systematic reviews. Clinical practice and clinical reasoning based on the response of these tests need to be re-evaluated in light of this. The reasons for the poor specificity include (i) an inability to selectively test the individual rotator cuff tissues, (ii) the presence of the subacromial bursa, which contains nociceptors and pain chemicals that will be stretched and compressed during all the clinical tests of the shoulder, and (iii) a lack of correlation between the current clinical tests and structural pathology identified with imaging in the form of ultrasound and magnetic resonance imaging.

\section{What this study adds}

Based on the lack of diagnostic accuracy of the clinical tests currently used, a new model of clinical assessment is proposed. The Shoulder Symptom Modification Procedure involves comparing symptomatic activities and movements identified by the patient before and after the application of a series of clinical techniques. The aim of this process is to find one or a combination of techniques that reduces the patient's symptoms. This proposal requires considerable research before it becomes an accepted clinical alternative.

The SSMP model is flexible and is capable of modification and evolution. As techniques that modify shoulder symptoms are identified, they may be incorporated into the model and procedures that are found to be less useful should be withdrawn.

\section{LIMITATIONS TO THIS MODEL}

Patients, health commissioning bodies and clinicians are embracing an evidence-based practice model of healthcare. ${ }^{48} 49$ Evidence-based practice (EBP) uses the most robust and appropriate research evidence available, in conjunction with the clinician's experiences and the patient's beliefs and values, ${ }^{48}{ }^{49}$ to inform practice. Where evidence is not available, the EBP model allows for clinical experience to guide practice. It is becoming increasingly evident that the special musculoskeletal tests used in current practice are little more than symptom provocation tests and, from an EBP perspective, their use to inform diagnosis is limited. ${ }^{1-4} 1450$ Although research evidence to justify the SSMP model is unavailable, EBP allows clinicians to propose models of assessment and management that are based on clinical experience with an understanding that research to verify or refute the clinical claims will follow. Research to investigate the usefulness of this model of assessment is being conducted and the utility of this model is its ability to change and adapt in response to new and emerging information.

In conclusion, current clinical tests used in the diagnosis of rotator cuff tendinopathy and subacromial impingement syndrome are of limited value due to their poor specificity. This suggests that they are useful as procedures that reproduce symptoms but not as a means of determining the origin of those symptoms. Patient management based on clinical reasoning derived from the findings of the currently recommended clinical 
orthopaedic tests may lack validity. The SSMP model assesses the individual or combined response to mechanical procedures performed around the shoulder, during aggravating movements. If individual or combined techniques improve symptoms, then similar techniques may be used in patient management. The Shoulder Symptom Modification Procedure is presented as one model of assessing the shoulder and informing patient management. Considerable research is required before this model should be accepted as a clinical alternative.

Competing interests: None declared.

Patient consent: Obtained.

\section{REFERENCES}

1. Lewis JS, Tennent TD. How effective are diagnostic tests for the assessment of rotator cuff disease of the shoulder? In: MacAuley D, Best TM, eds. Evidence-Based Sports Medicine. 2nd edn. London: Blackwell Publishing, 2007

2. Lewis JS, Valentine RE. The pectoralis minor length test: a study of the intra-rater reliability and diagnostic accuracy in subjects with and without shoulder symptoms. BMC Musculoskelet Disord 2007;8:64.

3. Fischer AM, Dexter WW. How evidence-based is our examination of the shoulder? In: MacAuley D, Best T, eds. Evidence-Based Sports Medicine. 2nd edn. Massachusetts: Blackwell Publishing - BMJ Books, 2007

4. Hegedus EJ, Goode A, Campbell S, et al. Physical examination tests of the shoulder: a systematic review with meta-analysis of individual tests. Br J Sports Med 2008;42:80-92; discussion 92

5. O'Brien SJ, Pagnani MJ, Fealy S, et al. The active compression test: a new and effective test for diagnosing labral tears and acromioclavicular joint abnormality. Am J Sports Med 1998;26:610-13

6. Laudner KG, Stanek JM, Meister K. Assessing posterior shoulder contracture: the reliability and validity of measuring glenohumeral joint horizontal adduction. J Athl Train 2006;41:375-80.

7. Jobe FW, Jobe CM. Painful athletic injuries of the shoulder. Clin Orthop Relat Res 1983;173:117-24.

8. Neer CS 2nd. Impingement lesions. Clin Orthop Relat Res 1983;(173):70-7.

9. Hawkins RJ, Kennedy JC. Impingement syndrome in athletes. Am J Sports Med 1980;8:151-8.

10. Zaslav KR. Internal rotation resistance strength test: a new diagnostic test to differentiate intra-articular pathology from outlet (Neer) impingement syndrome in the shoulder. J Shoulder Elbow Surg 2001;10:23-7.

11. Clark JM, Harryman DT 2nd. Tendons, ligaments, and capsule of the rotator cuff. Gross and microscopic anatomy. J Bone Joint Surg Am 1992;74:713-25.

12. Cyriax J, Cyriax P. Diagnosis of soft tissue lesions. 8th edn. London: Balliere Tindall, 1982.

13. Magee D. Orthopedic Physical Assessment. 3rd edn. Philadelphia: WB Saunders Co, 1997.

14. Miller CA, Forrester GA, Lewis JS. The validity of the lag signs in diagnosing fullthickness tears of the rotator cuff: a preliminary investigation. Arch Phys Med Rehabil 2008:89:1162-8.

15. Aszmann OC, Dellon AL, Birely BT, et al. Innervation of the human shoulder joint and its implications for surgery. Clin Orthop Relat Res 1996;(330):202-7.

16. Ide K, Shirai Y, Ito H. Sensory nerve supply in the human subacromial bursa. J Shoulder Elbow Surg 1996;5:371-82.

17. Gotoh M, Hamada K, Yamakawa H, et al. Interleukin-1-induced subacromial synovitis and shoulder pain in rotator cuff diseases. Rheumatology (Oxford) 2001:40:995-1001.

18. Sakai H, Fujita K, Sakai Y, et al. Immunolocalization of cytokines and growth factors in subacromial bursa of rotator cuff tear patients. Kobe J Med Sci 2001;47:25-34.

19. Yanagisawa K, Hamada K, Gotoh M, et al. Vascular endothelial growth factor (VEGF) expression in the subacromial bursa is increased in patients with impingement syndrome. J Orthop Res 2001;19:448-55.

20. Voloshin I, Gelinas J, Maloney MD, et al. Proinflammatory cytokines and metalloproteases are expressed in the subacromial bursa in patients with rotator cuff disease. Arthroscopy 2005:21:1076.

21. Frost $\mathbf{P}$, Andersen $\mathrm{JH}$, Lundorf $\mathrm{E}$. Is supraspinatus pathology as defined by magnetic resonance imaging associated with clinical sign of shoulder impingement? J Shoulder Elbow Surg 1999;8:565-8

22. Sher JS, Uribe JW, Posada A, et al. Abnormal findings on magnetic resonance images of asymptomatic shoulders. J Bone Joint Surg Am 1995:77:10-15.
23. Milgrom C, Schaffler M, Gilbert $S$, et al. Rotator-cuff changes in asymptomatic adults. The effect of age, hand dominance and gender. J Bone Joint Surg Br 1995; 77:296-8.

24. Miniaci A, Mascia AT, Salonen DC, et al. Magnetic resonance imaging of the shoulder in asymptomatic professional baseball pitchers. Am J Sports Med 2002:30:66-73.

25. Connor PM, Banks DM, Tyson AB, et al. Magnetic resonance imaging of the asymptomatic shoulder of overhead athletes: a 5-year follow-up study. Am J Sports Med 2003;31:724-7.

26. Worland RL, Lee D, Orozco CG, et al. Correlation of age, acromial morphology, and rotator cuff tear pathology diagnosed by ultrasound in asymptomatic patients. J South Orthop Assoc 2003;12:23-6.

27. Ben-Yishay A, Zuckerman JD, Gallagher M, et al. Pain inhibition of shoulder strength in patients with impingement syndrome. Orthopedics 1994;17:685-8.

28. Brox JI, Roe C, Saugen E, et al. Isometric abduction muscle activation in patients with rotator tendinosis of the shoulder. Arch Phys Med Rehabil 1997;78:1260-7.

29. Steenbrink F, de Groot JH, Veeger HE, et al. Pathological muscle activation patterns in patients with massive rotator cuff tears, with and without subacromial anaesthetics. Man Ther 2006;11:231-7.

30. Smidt N, Green S. Is the diagnosis important for the treatment of patients with shoulder complaints? Lancet 2003:362:1867-8.

31. Litaker D, Pioro M, El Bilbeisi $\mathrm{H}$, et al. Returning to the bedside: using the history and physical examination to identify rotator cuff tears. J Am Geriatr Soc 2000;48:1633-7.

32. Park HB, Yokota A, Gill HS, et al. Diagnostic accuracy of clinical tests for the different degrees of subacromial impingement syndrome. J Bone Joint Surg Am 2005:87:1446-55.

33. MacDonald PB, Clark P, Sutherland K. An analysis of the diagnostic accuracy of the Hawkins and Neer subacromial impingement signs. J Shoulder Elbow Surg 2000;9:299-301.

34. Lewis JS. My patient has non-specific shoulder pain. So what should I do now? In: South African Society of Physiotherapy National Biennial Congress: 22-25 September 2007; Durban, South Africa: 2007.

35. Lewis JS, Wright C, Green A. Subacromial impingement syndrome: the effect of changing posture on shoulder range of movement. J Orthop Sports Phys Ther 2005;35:72-87

36. Mulligan BR. Manual Therapy "Nags", "Snags", "MWMs" etc. 4th edn. New Zealand: Plane View Services, 1999

37. Maitland GD. Vertebral Manipulation. 5th edn. London: Butterworths, 1986

38. Zingg PO, Jost B, Sukthankar A, et al. Clinical and structural outcomes of nonoperative management of massive rotator cuff tears. J Bone Joint Surg Am 2007:89:1928-34.

39. Cholewinski JJ, Kusz DJ, Wojciechowski P, et al. Ultrasound measurement of rotator cuff thickness and acromio-humeral distance in the diagnosis of subacromial impingement syndrome of the shoulder. Knee Surg Sports Traumatol Arthrosc 2008;16:408-14.

40. Desmeules F, Minville L, Riederer B, et al. Acromio-humeral distance variation measured by ultrasonography and its association with the outcome of rehabilitation for shoulder impingement syndrome. Clin J Sport Med 2004;14:197-205.

41. Farrar JT, Young JP Jr, LaMoreaux L, et al. Clinical importance of changes in chronic pain intensity measured on an 11-point numerical pain rating scale. Pain 2001:94:149-58

42. Farrar JT, Berlin JA, Strom BL. Clinically important changes in acute pain outcome measures: a validation study. J Pain Symptom Manage 2003;25:406-11.

43. Bullock MP, Foster NE, Wright CC. Shoulder impingement: the effect of sitting posture on shoulder pain and range of motion. Man Ther 2005;10:28-37.

44. Teys $\mathbf{P}$, Bisset L, Vicenzino B. The initial effects of a Mulligan's mobilization with movement technique on range of movement and pressure pain threshold in painlimited shoulders. Man Ther 2008;13:37-42.

45. Boyle JJ. Is the pain and dysfunction of shoulder impingement lesion really second rib syndrome in disguise? Two case reports. Man Ther 1999;4:44-8.

46. Waddell G. The back pain revolution. 2nd edn. Edinburgh: Churchill Livingstone, 2004.

47. Lewis JS, Hewitt JS, Billington L, et al. A randomized clinical trial comparing two physiotherapy interventions for chronic low back pain. Spine 2005;30:711-21.

48. Sackett D, Straus S, Richardson W, et al. Evidence-based medicine. How to teach and practice EBM. 2nd edn. Edinburgh: Churchill Livingstone, 2000

49. Mayer D. Essential evidence-based medicine. Cambridge: Cambridge University Press, 2004.

50. Lewis JS, Green A, Wright C. Subacromial impingement syndrome: The role of posture and muscle imbalance. J Shoulder Elbow Surg 2005;14:385-92. 